\title{
Sfermion Precision Measurements at a Linear Collider*
}

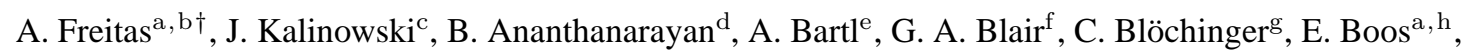
A. Brandenburg ${ }^{\mathrm{a}}$, A. Datta ${ }^{\mathrm{i}}$, A. Djouadi ${ }^{\mathrm{i}}$, H. Fraas ${ }^{\mathrm{g}}$, J. Guasch $^{\mathrm{j}}$, S. Hesselbach $^{\mathrm{e}}$, K. Hidaka ${ }^{\mathrm{k}}$, W. Hollik ${ }^{\mathrm{l}}$, T. Kernreiter ${ }^{\mathrm{e}}$, M. Maniatis ${ }^{\mathrm{a}}$, A. v. Manteuffel ${ }^{\mathrm{a}}$, H.-U. Martyn ${ }^{\mathrm{m}}$, D. J. Miller ${ }^{\mathrm{n}}$, G. Moortgat-Pick ${ }^{\mathrm{a}}$,

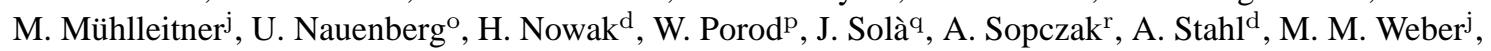
P. M. Zerwas ${ }^{\mathrm{a}}$

a Deutsches Elektronen-Synchrotron DESY, D-22603 Hamburg, Germany

${ }^{\mathrm{b}}$ Fermi National Accelerator Laboratory, Batavia, IL 60510-500, USA

${ }^{\mathrm{c}}$ Insitute of Theoretical Physics, Warsaw University, 00681 Warsaw, Poland

${ }^{\mathrm{d}}$ Deutsches Elektronen-Synchrotron DESY, D-15738 Zeuthen, Germany

eUniversität Wien, A-1090 Vienna, Austria

${ }^{\mathrm{f}}$ Royal Holloway and Bedford New College, University of London, UK

gUniversität Würzburg, D-97074 Würzburg, Germany

${ }^{\mathrm{h}}$ Moscow State University, 119899 Moscow, Russia

iUniversité de Montpellier II, F-34095 Montpellier Cedex 5, France

jPaul-Scherrer-Institute, CH-5232 Villigen PSI, Switzerland

${ }^{\mathrm{k}}$ Tokyo Gakugei University, Koganei, Tokyo 184-8501, Japan

${ }^{\text {l} M a x-P l a n c k-I n s t i t u t ~ f u ̈ r ~ P h y s i k, ~ D-80805 ~ M u ̈ n c h e n, ~ G e r m a n y ~}$

${ }^{\mathrm{m}}$ RWTH Aachen, D-52056 Aachen, Germany

${ }^{\mathrm{n}} \mathrm{CERN}, \mathrm{CH}-1211$ Genève 23, Switzerland

${ }^{\circ}$ University of Colorado, Boulder, CO, USA

pUniversität Zürich, CH-8057 Zürich, Switzerland

qUniversitat de Barcelona, E-08007 Barcelona, Spain

${ }^{\mathrm{r}}$ Lancaster University, Lancaster LA1 4YW, UK

At future $e^{ \pm} e^{-}$linear colliders, the event rates and clean signals of scalar fermion production-in particular for the scalar leptons - allow very precise measurements of their masses and couplings and the determination of their quantum numbers. Various methods are proposed for extracting these parameters from the data at the sfermion thresholds and in the continuum. At the same time, NLO radiative corrections and non-zero width effects have been calculated in order to match the experimental accuracy. The substantial mixing expected for the third generation sfermions opens up additional opportunities. Techniques are presented for determining potential CP-violating phases and for extracting $\tan \beta$ from the stau sector, in particular at high values. The consequences of possible large mass differences in the stop and sbottom system are explored in dedicated analyses.

\footnotetext{
* Based on talks given by A.F. at the 31st International Conference on High Energy Physics (ICHEP '02), Amsterdam, July 2002, and J.K at the International Workshop on Linear Colliders, Jeju Island,
}

Korea, August 2002 on behalf of the SUSY working groups of the ECFA/DESY and International Linear Collider Workshops; to appear in the proceedings.

†afreitas@fnal.gov 


\section{INTRODUCTION}

While the Standard Model of electroweak and strong interactions has been widely tested and stringently established in various experiments, it nevertheless exhibits conceptual drawbacks in the context of Grand Unified Theories and it does not include gravity. The introduction of supersymmetry provides a first step to resolve these problems. The Minimal Supersymmetric Standard Model (MSSM) comprises the minimal particle content necessary for extending the Standard Model into a supersymmetric theory. It is clear that supersymmetry is broken in nature, but the construction of a viable breaking mechanism remains a difficult issue. In most scenarios the breaking mechanism resides in an experimentally inaccessible "hidden sector" and is transmitted to the visible world, thus generating explicit soft-breaking terms in the effective Lagrangian.

If supersymmetric particles are detected in the future, exploring the underlying theory will include two central tasks. On the one hand, to establish symmetry scheme experimentally, it is necessary to accurately test the predicted symmetry relations, in particular the quantum numbers and couplings of the superpartners must coincide with the corresponding parameters of the Standard Model. On the other hand, the pattern of supersymmetry breaking needs to be explored. Because of the large number of soft-breaking parameters in the MSSM, this will be an enormous experimental effort. In this context, it is important to determine the soft-breaking parameters with high precision in order to reconstruct the underlying breaking mechanism, which eventually involves extrapolations to high energy scales. A high-energy $e^{+} e^{-}$ linear collider [1] is particularly suited for precision measurements of supersymmetric particle properties up to the per-mille level, due to the well-defined initial state, the high luminosity and the polarization for both beams [2]. In order to match the experimental accuracy, sufficiently precise and reliable theoretical predictions for the production cross-sections and decays of the superpartners are required.

This report is focused on recent developments in precision analyses of scalar fermions-sleptons and squarks-at a linear collider. In particular the determination of their quantum numbers, couplings and masses will be discussed, the latter being the essential

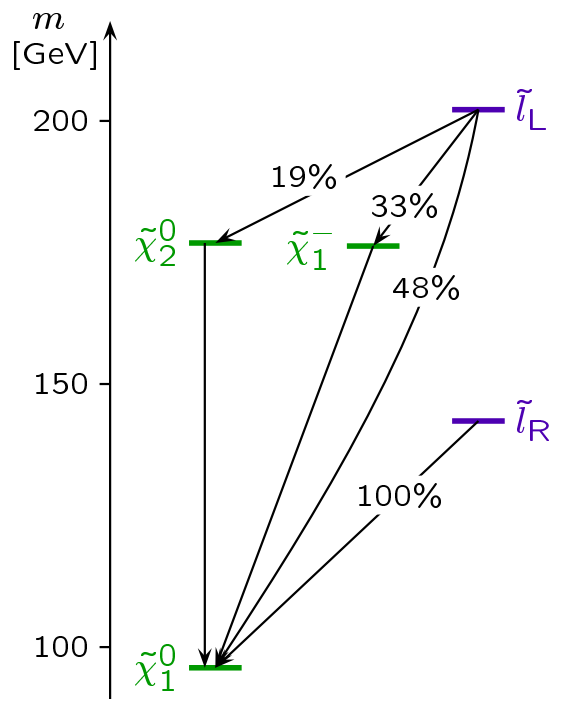

Figure 1. Masses and branching ratios of sleptons of the first two generations in the SPS1 scenario [3]. The total widths are $\Gamma_{\tilde{l}_{\mathrm{R}}}=210 \mathrm{MeV}$ and $\Gamma_{\tilde{l}_{\mathrm{L}}}=250 \mathrm{MeV}$.

building block for the exploration of the soft-breaking terms. Moreover, due to the large Higgs Yukawa coupling of the third generation sfermions, this sector can yield valuable information about the Higgs parameters $\mu$ and $\tan \beta$ as well as the trilinear scalar couplings $A_{\mathrm{f}}$, including potential CP-violating phases.

\section{DECAY AND PRODUCTION MODES}

The phenomenology of scalar leptons is characterized by clean final state signatures with only a few visible leptons and missing energy. The scalar partners $\tilde{f}_{\mathrm{L}}, \tilde{f}_{\mathrm{R}}$ of the left- and right-handed states of the fermion $f$ have different decay modes which can be used to discriminate between the two chirality states:

$$
\begin{aligned}
\tilde{f}_{\mathrm{R}}^{-} \rightarrow f^{-} \tilde{\chi}_{1}^{0}, \quad \tilde{f}_{\mathrm{L}}^{-} \rightarrow f^{-} \tilde{\chi}_{2}^{0} & \stackrel{\llcorner}{\longrightarrow} \tau^{+} \tau^{-} \tilde{\chi}_{1}^{0} \\
\tilde{f}_{\mathrm{L}}^{-} \rightarrow f^{\prime-} \tilde{\chi}_{1}^{-} & \stackrel{L}{\longrightarrow} \tau^{-} \nu_{\tau} \tilde{\chi}_{1}^{0}
\end{aligned}
$$

where $f^{\prime}$ is the $\mathrm{SU}(2)$ doublet partner of $f$. Fig. 1 shows the mass spectrum and branching ratios of the first and second generation sleptons for the SPS1 scenario [3]. 
(a)

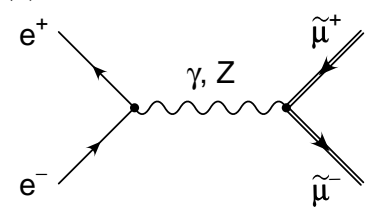

(b)

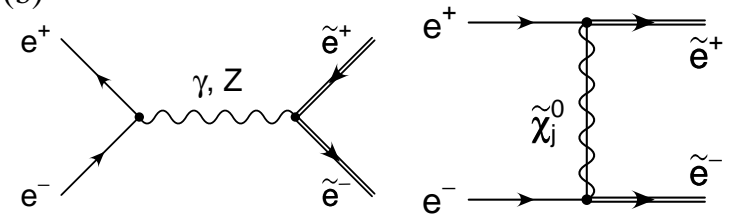

(c)

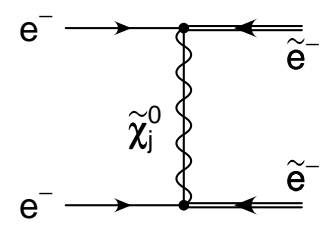

Figure 2. Tree-level diagrams for the production of smuons (a) and selectrons (b) in $e^{+} e^{-}$annihilation and for the production of selectrons in $e^{-} e^{-}$collisions (c).

At leading order, sleptons of the second and third generation, as well as squarks, are produced via schannel gauge-boson exchange in $e^{+} e^{-}$annihilation, see Fig. 2 (a). As a consequence, they are produced in a P-wave with a characteristic rise of the excitation curve $\sigma \propto \beta^{3}$, where $\beta=\sqrt{1-4 m_{\tilde{\mathrm{f}}}^{2} / s}$ is the sfermion velocity. Due to the Majorana nature of the neutralinos in the t-channel, see Fig. 2 (b) and (c), selectrons can also be produced in S-waves $(\sigma \propto \beta)$, namely for $\tilde{e}_{\mathrm{R}}^{ \pm} \tilde{e}_{\mathrm{L}}^{\mp}$ pairs in $e^{+} e^{-}$annihilation and $\tilde{e}_{\mathrm{R}}^{-} \tilde{e}_{\mathrm{R}}^{-}, \tilde{e}_{\mathrm{L}}^{-} \tilde{e}_{\mathrm{L}}^{-}$pairs in $e^{-} e^{-}$scattering. The $e^{-} e^{-}$mode has the additional advantage of much lower background.

\section{SELECTRON QUANTUM NUMBERS}

Due to the small masses of the first two generation fermions, the mixing between the corresponding scalar partners is expected to be negligible. As a consequence, the mass eigenstates $\tilde{f}_{1}, \tilde{f}_{2}$ are almost identical to the chirality states $\tilde{f}_{\mathrm{L}}, \tilde{f}_{\mathrm{R}}$, which are the scalar partners of the left- and right-handed states of the fermion $f$, respectively.

The additional t-channel amplitude for selectron production directly relates the quantum numbers of the produced selectrons and spositrons to the polarization of the incoming electron and positron. Therefore the chiral quantum numbers of the selectrons can be unambiguously determined by studying the selectron production cross-sections in $e^{+} e^{-}$annihilation with polarized beams [4]. In order to separate the t-channel neutralino exchange from the s-channel photon and Z-boson exchange, both the electron and positron beams must be polarized. By comparing the selectron cross-section for different polarization combinations the chiral quantum numbers of the selectrons can be disentangled.

\section{SUSY COUPLING RELATIONS}

One of the most fundamental predictions of supersymmetry is the equivalence between gauge couplings and their supersymmetric Yukawa counterparts. For instance, the gauge coupling between a vector boson $V$ and a fermion $f, g(V f f)$, is related to the Yukawa coupling between the gaugino partner $\tilde{V}$ of the vector boson, the fermion $f$ and the sfermion $\tilde{f}$, $\hat{g}(\tilde{V} f \tilde{f})$. Within softly broken supersymmetric theories both kinds of couplings are required to be identical,

$g=\hat{g}$.

This relation can be tested in the electroweak sector by measuring the production cross-sections of scalar leptons. The Yukawa couplings $\hat{g}$ can best be probed in the production of selectrons, as a result of the tchannel neutralino exchange contributions [5]. One can take advantage of the $e^{-} e^{-}$mode due to reduced background, larger cross-sections, higher polarizability and no interfering s-channel contributions [6]. Simulations have shown that these couplings can be determined with high accuracy by measuring the slepton cross-sections at a linear collider [8,9], see Tab. 1. This method is complementary to the measurement of the Yukawa couplings in chargino and neutralino pair production [7]. 
Table 1

Expected errors for the extraction of the supersymmetric Yukawa couplings $\hat{g}^{\prime}$ and $\hat{g}$, which correspond to the $\mathrm{U}(1)_{\mathrm{Y}}$ and $\mathrm{SU}(2)_{\mathrm{L}}$ gauge couplings $g^{\prime}$ and $g$, respectively. No detector simulation is included. The values are for the SPS1 scenario [3] and $\sqrt{s}=500 \mathrm{GeV}$.

\begin{tabular}{lrr}
\hline Process & Luminosity & Result \\
\hline$e^{+} e^{-} \rightarrow\left(\tilde{e}_{\mathrm{R}}^{+} \tilde{e}_{\mathrm{R}}^{-}\right) \rightarrow e^{+} e^{-} \tilde{\chi}_{1}^{0} \tilde{\chi}_{1}^{0}$ & $500 \mathrm{fb}^{-1}$ & $\delta \hat{g}^{\prime} / \hat{g}^{\prime} \approx 0.2 \%$ \\
$e^{+} e^{-} \rightarrow\left(\tilde{e}_{\mathrm{R}}^{ \pm} \tilde{e}_{\mathrm{L}}^{+}\right) \rightarrow e^{+} e^{-} \tilde{\chi}_{1}^{0} \tilde{\chi}_{2}^{0} \rightarrow e^{+} e^{-} \tau \tau \tilde{\chi}_{1}^{0} \tilde{\chi}_{1}^{0}$ & & $\delta \hat{g} / \hat{g} \approx 0.7 \%$ \\
\hline$e^{-} e^{-} \rightarrow\left(\tilde{e}_{\mathrm{R}}^{-} \tilde{e}_{\mathrm{R}}^{-}\right) \rightarrow e^{-} e^{-} \tilde{\chi}_{1}^{0} \tilde{\chi}_{1}^{0}$ & $50 \mathrm{fb}^{-1}$ & $\delta \hat{g}^{\prime} / \hat{g}^{\prime} \approx 0.2 \%$ \\
$e^{-} e^{-} \rightarrow\left(\tilde{e}_{\mathrm{L}}^{-} \tilde{e}_{\mathrm{L}}^{-}\left(\rightarrow e^{+} e^{-} \tilde{\chi}_{2}^{0} \tilde{\chi}_{2}^{0} \rightarrow e^{+} e^{-} 4 \tau \tilde{\chi}_{1}^{0} \tilde{\chi}_{1}^{0}\right.\right.$ & & $\delta \hat{g} / \hat{g} \approx 0.8 \%$ \\
\hline
\end{tabular}

Clearly the experimental precision requires the inclusion of radiative corrections in the theoretical predictions for the slepton cross-sections. As a first step the produced sleptons may be considered on-shell, since in the continuum far above threshold the effect of the non-zero slepton width is relatively small, of the order $\Gamma_{\tilde{f}} / m_{\tilde{f}}$. As a consequence, the production and decay of the sleptons can be treated separately. For both sub-processes, the complete electroweak one-loop corrections in the MSSM have been computed [10,11]. In Ref. [10] the pair production of smuons in $e^{+} e^{-}$and the production of selectrons in $e^{+} e^{-}$and $e^{-} e^{-}$collisions have been calculated at next-to-leading order. The $\mathcal{O}(\alpha)$ corrections for the most important decay channels of the sleptons, $\tilde{l}^{ \pm} \rightarrow l^{ \pm} \tilde{\chi}_{i}^{0}$ and $\tilde{l}^{ \pm} \rightarrow\left(\bar{\nu}_{l}\right) \tilde{\chi}_{i}^{ \pm}$have been considered in Ref. [11].

Both calculations have been performed in the onshell renormalization scheme. While the renormalization of the gauge couplings and gauge bosons can be performed similar to the Standard Model case, the renormalization of the superpartner contributions is slightly more involved. For example, the sector of the charginos and neutralinos depends on the gaugino parameters $M_{1}, M_{2}$ and the Higgs parameters $\mu$ and $\tan \beta$. It should be noted that the definition of $\tan \beta$ cannot be related to any physical observable in a straightforward manner [12]. Instead, for technical reasons, the $\overline{\mathrm{DR}}$ scheme is used in [10] for the renormalization of $\tan \beta$. By demanding on-shell mass conditions for three out of the six charginos and neutralinos, the parameters $M_{1}, M_{2}$ and $\mu$ can be fixed. The other three mass eigenvalues then receive nonzero corrections at the one-loop level. For more details on the renormalization see $[8,10,11]$.

The effects of the electroweak corrections were found to be sizeable, of the order of 5-10\%. They include important effects from supersymmetric particles in the virtual corrections, in particular nondecoupling logarithmic contributions, e.g. terms $\propto$ $\log m_{\tilde{f}} / m_{\text {weak }}$ from fermion-sfermion-loops.

The equality of gauge and Yukawa couplings in the $\mathrm{SU}(3)_{\mathrm{C}}$ gauge sector can be tested at a linear collider by investigating the associated production of quarks $q$ and squarks $\tilde{q}$ with a gluon $g$ or gluino $\tilde{g}$. While the processes $e^{+} e^{-} \rightarrow q \bar{q} g$ and $e^{+} e^{-} \rightarrow \tilde{q} \tilde{\tilde{q}} g$ are sensitive to the strong gauge coupling of quarks and squarks, respectively, the corresponding Yukawa coupling can be probed in $e^{+} e^{-} \rightarrow q \overline{\tilde{q}} \tilde{g}$. In order to obtain reliable theoretical predictions for these crosssections it is necessary to include next-to-leading order (NLO) supersymmetric QCD corrections. These corrections are generally expected to be rather large and they are necessary to reduce the large scale dependence of the leading-order result. The NLO QCD corrections to the process $e^{+} e^{-} \rightarrow q \bar{q} g$ within the Standard Model are known since long [13]. Recently, the complete $\mathcal{O}\left(\alpha_{\mathrm{s}}\right)$ corrections to all three processes in the MSSM have been calculated [14]. The NLO contributions enhance the cross-section in the peak region by roughly $20 \%$ with respect to the LO result. Furthermore, the scale dependence is reduced by a factor of about six when the NLO corrections are included.

\section{MASS DETERMINATION}

In models with R-parity conservation, the mass of supersymmetric particles cannot directly be reconstructed from its decay products since the lightest supersymmetry particle (LSP) - mostly the lightest neutralino $\tilde{\chi}_{1}^{0}$ - escapes undetected. Nevertheless both the mass of scalar fermions and the unobserved LSP can be determined by measuring the end points of 
the kinematical energy distributions of the visible decay fermions $[15,16]$. However the efficiency of this method is affected by initial-state radiation (ISR) and beamstrahlung effects and cuts necessary to reduce the backgrounds.

A novel approach for determining the selectron masses [17] takes advantage of the fact that selectrons can be produced in mixed pairs, $e^{+} e^{-} \rightarrow \tilde{e}_{\mathrm{R}}^{+} \tilde{e}_{\mathrm{L}}^{-}$, $\tilde{e}_{\mathrm{L}}^{+} \tilde{e}_{\mathrm{R}}^{-}$. Because of the mass difference between Land R-selectrons, the minima and maxima of the final state lepton energy distributions originating from an $\tilde{e}_{\mathrm{R}}^{+} \tilde{e}_{\mathrm{L}}^{-}$pair will be different for the final electron and positron. When subtracting the normalized electron and positron distributions, the relevant kinematical edges will therefore remain visible while the Standard Model background and a large part of supersymmetric backgrounds are canceled. In particular, the contributions from the production of selectrons with same chirality, $\tilde{e}_{\mathrm{R}}^{+} \tilde{e}_{\mathrm{R}}^{-}$and $\tilde{e}_{\mathrm{L}}^{+} \tilde{e}_{\mathrm{L}}^{-}$, are cancelled while the signal from mixed pairs, $\tilde{e}_{\mathrm{R}}^{+} \tilde{e}_{\mathrm{L}}^{-}$and $\tilde{e}_{\mathrm{L}}^{+} \tilde{e}_{\mathrm{R}}^{-}$is preserved. In order to disentangle $\tilde{e}_{\mathrm{R}}^{+} \tilde{e}_{\mathrm{L}}^{-}$pairs from $\tilde{e}_{\mathrm{L}}^{+} \tilde{e}_{\mathrm{R}}^{-}$pairs, appropriate polarization of the incident electron beam is used and the subtracted electron and positron distributions for left- and right-handed $e^{-}$beam polarization are again subtracted. The final distribution including ISR and beamstrahlung and cascade decays shown in Fig. 3 nicely exhibits the characteristic kinematical edges while being unaffected by backgrounds. With this method it is possible to determine the masses of the selectrons and the neutralino LSP with a precision of $200-500 \mathrm{MeV}$ for an integrated luminosity of $\int L=1000 \mathrm{fb}^{-1}$.

Alternatively, the sfermion masses can be extracted from the measurement of the pair production cross-sections near threshold. The characteristic onset of the excitation curve allows a very precise determination of the sfermion masses in threshold scans. Since the expected experimental accuracy is of $\mathcal{O}(100 \mathrm{MeV})$, it is necessary to incorporate effects beyond leading order in the theoretical predictions [18]. The non-zero widths of the sfermions, which considerably affect the cross-sections near threshold, must be included in a gauge-invariant manner. This can be achieved by shifting the sfermion mass into the complex plane, $m_{\tilde{\mathrm{f}}}^{2} \rightarrow m_{\tilde{\mathrm{f}}}^{2}-i m_{\tilde{\mathrm{f}}} \Gamma_{\tilde{f}}$. Moreover, for the production of off-shell sfermions, the full $2 \rightarrow 4$ matrix element (e.g. $e^{+} e^{-} \rightarrow f^{+} f^{-} \tilde{\chi}_{1}^{0} \tilde{\chi}_{1}^{0}$ ) must be taken into account, including the decay of the

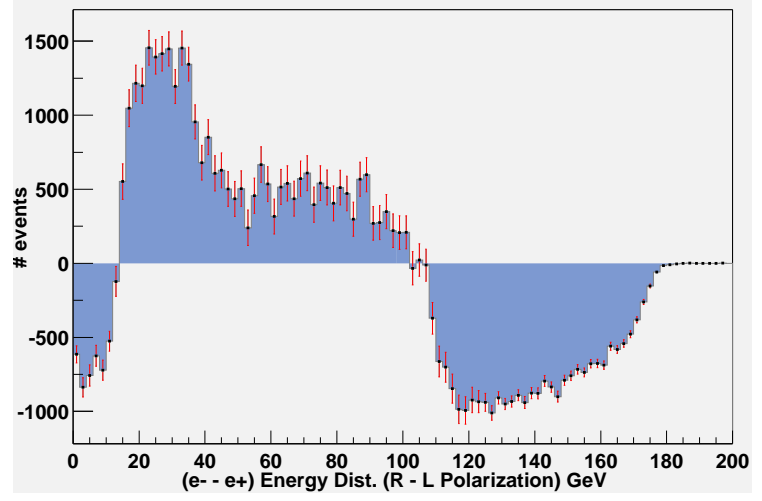

Figure 3. Difference of the subtracted normalized electron and positron energy distributions for $80 \% \mathrm{~L}$ and R-polarized $e^{-}$beam.

sfermions as well as the MSSM backgrounds and interference contributions. One of the most important radiative corrections near threshold is the Coulomb rescattering correction due to photon exchange between the slowly moving sfermions. For the production of off-shell sfermions with orbital angular momentum $l$ it is given by

$$
\begin{aligned}
\sigma_{\text {coul }}= & \sigma_{\text {born }} \frac{\alpha \pi}{2 \beta_{p}} Q_{\tilde{\mathrm{f}}}^{2} \\
& \times\left[1-\frac{2}{\pi} \arctan \frac{\left|\beta_{M}\right|^{2}-\beta_{p}^{2}}{2 \beta_{p} \Im m \beta_{M}}\right] \Re e \mathcal{C}_{l}, \\
\mathcal{C}_{l}= & {\left[\frac{\beta_{p}^{2}+\beta_{M}^{2}}{2 \beta_{p}^{2}}\right]^{l} }
\end{aligned}
$$

with

$$
\begin{aligned}
\beta_{p} & =\frac{1}{s} \sqrt{\left(s-p_{+}^{2}-p_{-}^{2}\right)^{2}-4 p_{+}^{2} p_{-}^{2}}, \\
\beta_{M} & =\frac{1}{s} \sqrt{\left(s-M_{+}^{2}-M_{-}^{2}\right)^{2}-4 M_{+}^{2} M_{-}^{2}} .
\end{aligned}
$$

$Q_{\tilde{\mathrm{f}}}, p_{ \pm}$and $M_{ \pm}^{2}=m_{ \pm}^{2}-i m_{ \pm} \Gamma_{ \pm}$are the charge quantum number, the momenta and complex pole masses of the produced sfermion and anti-sfermion. Beamstrahlung and ISR also play an important role. As mentioned in sec. 2 , selectrons can in addition be produced in $e^{-} e^{-}$collisions.

As an example, the selectron cross-sections for both collider modes and the cross-section for smuon production in $e^{+} e^{-}$annihilation are shown in Fig. 4 

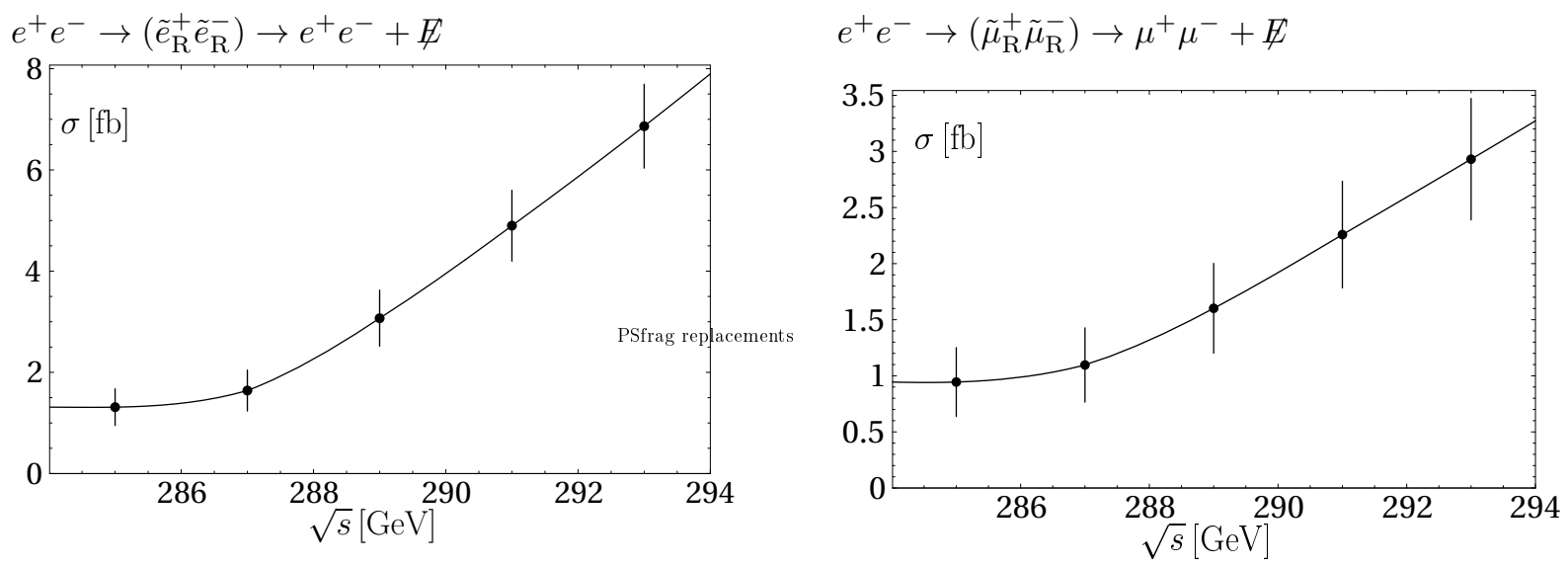

$e^{-} e^{-} \rightarrow\left(\tilde{e}_{\mathrm{R}}^{-} \tilde{e}_{\mathrm{R}}^{-}\right) \rightarrow e^{-} e^{-}+\not E$

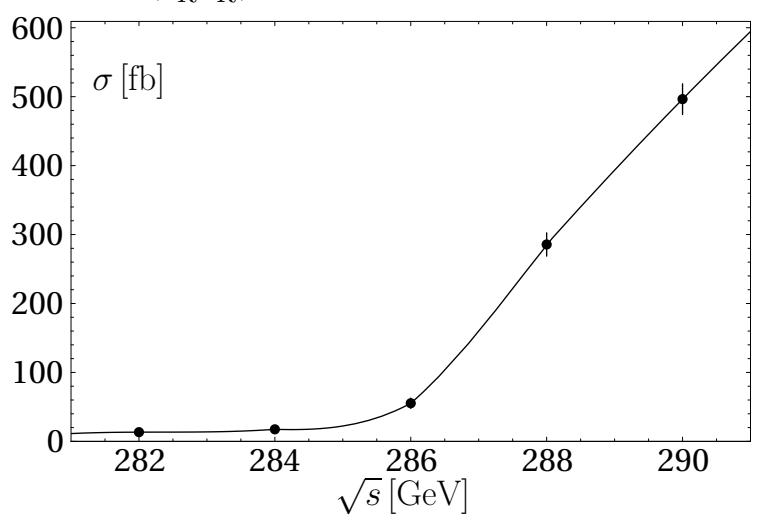

Figure 5. Threshold excitation curve for R-smuon pair production with error bars corresponding to $\int L=10 \mathrm{fb}^{-1}$ per scan point (SPS1 scenario).

for reconstructing the soft-breaking parameters at the GUT and Planck scales [19]. As an example, in [20] a method is proposed to probe a non-universality between the left and right slepton masses at the GUT scale if only both selectrons and the light chargino are accessible at a linear collider. Inspired by the oneloop RGE for slepton masses in mSUGRA-type models the quantity

Figure 4. Threshold excitation curves for R-selectron pair production. The error bars indicate the statistical error for a luminosity per scan point of $\int L=10 \mathrm{fb}^{-1}$ in $e^{+} e^{-}$and $\int L=1 \mathrm{fb}^{-1}$ in $e^{-} e^{-}$mode, respectively (SPS1 scenario).

and Fig. 5, including five equidistant scan points. Using four free parameters, the mass, width, normalization and a flat background contribution, it is possible to fit the excitation cross-section in a modelindependent way. Results for the production of selectrons are given in Tab. 2. The numbers have been obtained with a MC generator without detector simulation but including ISR and the theoretical refinements mentioned above. The backgrounds from both SM and MSSM sources, reduced by appropriate cuts, have also been included.

These high-precision measurements are necessary

is defined which is strongly correlated with the slepton mass non-universality $\delta m^{2} \equiv m_{\tilde{e}_{R}}^{2}\left(M_{\mathrm{GUT}}\right)-$ $m_{\tilde{e}_{L}}^{2}\left(M_{\mathrm{GUT}}\right)$. The method allows to detect a nonuniversality if $\left|\delta m^{2}\right| \gtrsim 5 \cdot 10^{3} \mathrm{GeV}^{2}$. Using additional information on $M_{2}$ this improves to $\left|\delta m^{2}\right| \gtrsim 2$. $10^{3} \mathrm{GeV}^{2}$, i.e. $\delta m / m \gtrsim 1.6 \%$ for $m \approx 250 \mathrm{GeV}$.

\section{SINGLE SLEPTON PRODUCTION}

In general, only sfermions with masses up to the beam energy, $m_{\tilde{\mathrm{f}}}<\sqrt{s} / 2$, can be investigated using the pair production process. Below the pair production threshold, sfermions can only be produced in association with their partner fermions and charginos or neutralinos. With sufficiently high luminosity at 
Table 2

Expected precision for the determination of selectron masses and widths from threshold scans [8,9]. The values are for the SPS1 scenario.

\begin{tabular}{|c|c|c|c|}
\hline Process & $\begin{array}{l}\text { umn. } \\
\left.b^{-1}\right]\end{array}$ & $\begin{array}{l}\text { Mass } \\
{[\mathrm{GeV}]}\end{array}$ & $\begin{array}{r}\text { Width } \\
{[\mathrm{MeV}]}\end{array}$ \\
\hline $\begin{aligned} e^{+} e^{-} & \rightarrow\left(\tilde{e}_{\mathrm{R}}^{+} \tilde{e}_{\mathrm{R}}^{-}\right) \\
& \rightarrow e^{+} e^{-}+\not E\end{aligned}$ & $5 \times 10$ & $\begin{array}{c}m_{\tilde{e}_{R}} \\
143.0_{-0.19}^{+0.21}\end{array}$ & $\begin{array}{r}\Gamma_{\tilde{e}_{R}} \\
150_{-250}^{+300}\end{array}$ \\
\hline $\begin{aligned} e^{-} e^{-} & \rightarrow\left(\tilde{e}_{\mathrm{R}}^{-} \tilde{e}_{\mathrm{R}}^{-}\right) \\
& \rightarrow e^{-} e^{-}+E\end{aligned}$ & $5 \times 1$ & $142.95_{-0.053}^{+0.048}$ & $200_{-}^{+}$ \\
\hline $\begin{aligned} e^{+} e^{-} & \rightarrow\left(\tilde{e}_{\mathrm{R}}^{ \pm} \tilde{e}_{\mathrm{L}}^{ \pm}\right) \\
& \rightarrow e^{+} e^{-} \tau \tau+\end{aligned}$ & $5 \times 10$ & $\begin{array}{c}m_{\tilde{e}_{\mathrm{L}}} \\
202.2_{-0.33}^{+0.37}\end{array}$ & $\begin{array}{c}\Gamma_{\tilde{e}_{\mathrm{L}}} \\
240_{-40}^{+40}\end{array}$ \\
\hline $\begin{aligned} e^{-} e^{-} & \rightarrow\left(\tilde{e}_{\mathrm{L}}^{-} \tilde{e}_{\mathrm{L}}^{-}\right) \\
& \rightarrow e^{-} e^{-} 4 \tau\end{aligned}$ & $5 \times 1$ & $202.2_{-0.44}^{+0.62}$ & $240-4$ \\
\hline
\end{tabular}

a future linear collider $\left(\int L=500 \mathrm{fb}^{-1}\right)$, it is possible to probe sfermions with masses that exceed the beam energy by a few tens of $\mathrm{GeV}$ if the gauginos are relatively light [21]. Due to the additional photon exchange in the t-channel, the discovery potential of the associated production is particularly promising for selectrons.

\section{THIRD GENERATION SFERMIONS}

In contrast to the first two generation sfermions, large mixings are expected between the left- and right-chiral states of the third generation sfermions due to the large Yukawa coupling. It can be seen from the sfermion mass matrix that for large fermion masses $m_{\mathrm{f}}$ the sensitivity to the Higgs parameters $\mu$ and $\tan \beta$ as well as the trilinear scalar couplings $A_{\mathrm{f}}$ is enhanced:

$M_{\tilde{\mathrm{f}}}^{2}=\left(\begin{array}{cc}m_{\mathrm{f}}^{2}+m_{\tilde{\mathrm{F}}_{\mathrm{L}}}^{2}+D_{\mathrm{L}} & m_{\mathrm{f}}\left(A_{\mathrm{f}}-\mu / t_{\beta}^{2 I_{\mathrm{f}}^{3}}\right) \\ m_{\mathrm{f}}\left(A_{\mathrm{f}}-\mu / t_{\beta}^{2 I_{\mathrm{f}}^{3}}\right) & m_{\mathrm{f}}^{2}+m_{\tilde{\mathrm{f}}_{\mathrm{R}}}^{2}+D_{\mathrm{R}}\end{array}\right)$,

where $m_{\tilde{\mathrm{F}}_{\mathrm{L}}}, m_{\tilde{\mathrm{f}}_{\mathrm{R}}}, D_{\mathrm{L}}, D_{\mathrm{R}}$ are the soft-breaking masses and D-terms of the sfermions $\tilde{f}_{\mathrm{L}, \mathrm{R}}$, and $I_{\mathrm{f}}^{3}$ is the weak isospin quantum number of the fermion $f$. The shortcut $t_{\beta} \equiv \tan \beta$ has been used.

For instance, the production and decay of staus is interesting for the determination of $\tan \beta$, in particu-

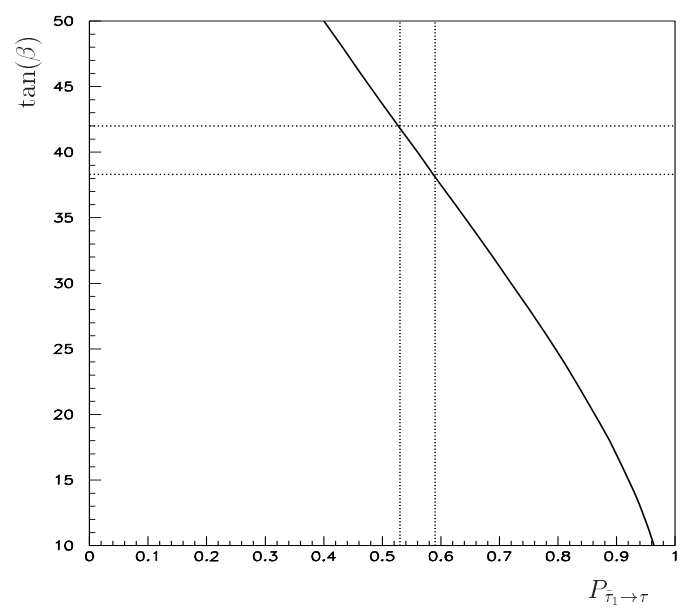

Figure 6. Determination of $\tan \beta$ from the tau polarization $P_{\tilde{\tau}_{1} \rightarrow \tau}$ in the decay $\tilde{\tau}_{1}^{-} \rightarrow \tau^{-} \tilde{\chi}_{1}^{0}$.

lar at high values ${ }^{3}$, by examining the polarization of the taus in the decay $\tilde{\tau}_{1,2}^{-} \rightarrow \tau_{\mathrm{L} . \mathrm{R}}^{-} \tilde{\chi}_{1}^{0}[22,5]$. In a recent study within the MSSM [23] it was found that $\tan \beta$ in the range of 30-40 can be determined with an error of about $10 \%$ when the $\tau$ polarization is measured only for $\tilde{\tau}_{1}$ decays (see also Fig. 6), assuming a sufficiently large higgsino component of the lightest neutralino $\tilde{\chi}_{1}^{0}$.

The parameters $\mu$ and $A_{\tau}$ as well as the neutralino parameter $M_{1}$ may carry $\mathrm{CP}$-violating phases. By using measurements of stau masses, cross-sections and branching ratios, it is possible to extract these parameters including their phases from the stau system. Very high accuracy on all involved parameters can be obtained [24] if the decay of the heavy staus into heavy Higgs bosons is kinematically allowed, see Tab. 3 .

$\mathrm{CP}$-violating parameters in the stau sector can also give rise to electric and weak dipole moments of the taus. At a linear collider with high luminosity and polarization of both $e^{+}$and $e^{-}$beams it is possible to probe $\mathrm{CP}$-violating tau dipole form factors up to the level of $\mathcal{O}(3)-\mathcal{O}(5) \cdot 10^{-19} e \mathrm{~cm}$ [25]. While this precision would improve the current experimental bounds by three orders of magnitude, it is however still about one order of magnitude beyond the expectations from supersymmetric models with $\mathrm{CP}$ -

${ }^{3}$ In this region the chargino/neutralino analyses are not effective [7]. 
Table 3

Extracted parameters from simulated data of the masses, cross-sections and BRs of $\tilde{\tau}_{i}$ for $\sqrt{s}=$ $800 \mathrm{GeV}$ and $\int L=500 \mathrm{fb}^{-1}$. Original values: $M_{\tilde{\tau}_{\mathrm{L}}}=350 \mathrm{GeV}, m_{\tilde{\tau}_{\mathrm{R}}}=150 \mathrm{GeV}, A_{\tau}=$ $-800 i \mathrm{GeV}, M_{2}=280 \mathrm{GeV}, \mu=250 \mathrm{GeV}$, $\phi_{\mathrm{U}(1)}=0$.

\begin{tabular}{lcc}
\hline $\tan \beta$ & 3 & 30 \\
\hline$m_{\tilde{\tau}_{\mathrm{R}}}^{2}\left[\mathrm{GeV}^{2}\right]$ & $(225 \pm 2) \cdot 10^{2}$ & $(225 \pm 6) \cdot 10^{2}$ \\
$m_{\tilde{\tau}_{\mathrm{L}}}^{2}\left[\mathrm{GeV}^{2}\right]$ & $(1225 \pm 4) \cdot 10^{2}$ & $(1229 \pm 7) \cdot 10^{2}$ \\
$\Re e A_{\tau}[\mathrm{GeV}]$ & $-8 \pm 180$ & $8 \pm 55$ \\
$\Im m A_{\tau}[\mathrm{GeV}]$ & $-800 \pm 70$ & $-800 \pm 21$ \\
\hline$\Re e \mu[\mathrm{GeV}]$ & $249.9 \pm 0.26$ & $249.9 \pm 0.6$ \\
$\Im m \mu[\mathrm{GeV}]$ & $2.4 \pm 1.7$ & $-0.2 \pm 3.8$ \\
$\tan \beta$ & $2.99 \pm 0.03$ & $29.9 \pm 0.7$ \\
$\Re e M_{1}[\mathrm{GeV}]$ & $140.9 \pm 0.2$ & $140.6 \pm 0.6$ \\
$\Im m M_{1}[\mathrm{GeV}]$ & $-0.7 \pm 3.4$ & $0.16 \pm 1.0$ \\
$M_{2}[\mathrm{GeV}]$ & $280.0 \pm 0.3$ & $280 \pm 1$ \\
\hline
\end{tabular}

violation.

The study of scalar top quarks is of particular interest since due to large mixing the lighter stop mass eigenstate is likely to be the lightest squark. As a consequence, the decay channel into $t \tilde{\chi}_{1}^{0}$ may be kinematically forbidden so that other dedicated search strategies are necessary. The most promising decay modes in this case are $\tilde{t}_{1} \rightarrow c \tilde{\chi}_{1}^{0}$ and $\tilde{t}_{1} \rightarrow b \tilde{\chi}_{1}^{ \pm}$[26]. A simulation including full-statistics SM backgrounds and a detailed investigation of quark tagging and supersymmetric backgrounds for the SPS5 scenario ${ }^{4}$ [3] has been performed [27]. Using the decay channel $\tilde{t}_{1} \rightarrow c \tilde{\chi}_{1}^{0}$, the mass of the lighter stop and the mixing angle can be constrained up to $\Delta m_{\tilde{\mathrm{t}}_{1}} \approx 1 \mathrm{GeV}$ and $\Delta \cos \theta_{\tilde{t}_{1}} \approx 0.0125$ with a luminosity of 1000 $\mathrm{fb}^{-1}$, while other decay channels like $\tilde{t}_{1} \rightarrow b \tilde{\chi}_{1}^{ \pm}$and $\tilde{t}_{1} \rightarrow b l \tilde{\nu}_{l}$ are not open in this specific scenario.

For stops and sbottoms with large mass differences, bosonic decay modes, $\tilde{t}_{1} \rightarrow \tilde{b}_{1}+\left(H^{+}\right.$or $\left.W^{+}\right)$, $\tilde{b}_{1} \rightarrow \tilde{t}_{1}+\left(H^{-}\right.$or $\left.W^{-}\right)$, may become important. Due to the large Yukawa couplings and mixings of $\tilde{t}$ and $\tilde{b}$ these decay modes can dominate in a wide

\footnotetext{
${ }^{4}$ The SPS5 scenario is a dedicated "light-stop" scenario
}

range of MSSM parameters [28]. Furthermore the bosonic decay modes are affected by large radiative SUSY-QCD corrections [29], whose leading contribution can be taken into account by SUSY-QCD running parameters for the stop/sbottom masses and the trilinear couplings. As a consequence, the precise investigation of these decay modes is important for stop/sbottom searches and the determination of the underlying MSSM parametersat a future linear collider.

Acknowledgements: This work was supported in part by the European Commision 5th framework under contract HPRN-CT-2000-00149 and the PolishGerman LC project No. POL 00/015. W. P. is supported by the "Erwin Schrödinger fellowship No. J2095" of the "Fonds zur Förderung der wissenschaftlichen Forschung" of Austria FWF and partly by the Swiss "Nationalfonds".

\section{REFERENCES}

1. Tesla Technical Design Report, Part III, eds. R. Heuer, D. J. Miller, F. Richard and P. M. Zerwas, DESY-2001-11C, [hep-ph/ 0106315]; T. Abe et al. [American Linear Collider Working Group Collaboration], in Proc. of the APS/DPF/DPB Summer Study on the Future of Particle Physics (Snowmass 2001) eds. R. Davidson and C. Quigg, SLAC-R-570, [hep-ex/0106056]; K. Abe et al. [ACFA Linear Collider Working Group Collaboration], KEKREPORT-2001-11, [hep-ph/0109166].

2. G. Moortgat-Pick, these proceedings [hep$\mathrm{ph} / 0210212]$.

3. B. C. Allanach et al., in Proc. of the APS/DPF/DPB Summer Study on the Future of Particle Physics (Snowmass 2001) eds. R. Davidson and C. Quigg, Eur. Phys. J. C 25 (2002) 113. N. Ghodbane and H. U. Martyn, in Proc. of the APS/DPF/DPB Summer Study on the Future of Particle Physics (Snowmass 2001) eds. R. Davidson and C. Quigg, [hep-ph/0201233].

4. C. Blöchinger, H. Fraas, G. Moortgat-Pick and W. Porod, Eur. Phys. J. C 24 (2002) 297.

5. M. M. Nojiri, K. Fujii and T. Tsukamoto, Phys. Rev. D 54 (1996) 6756.

6. H. C. Cheng, J. L. Feng and N. Polonsky, Phys. 
Rev. D 57 (1998) 152.

7. S. Y. Choi, A. Djouadi, M. Guchait, J. Kalinowski, H. S. Song and P. M. Zerwas, Eur. Phys. J. C 14 (2000) 535; S. Y. Choi, J. Kalinowski, G. Moortgat-Pick and P. M. Zerwas, Eur. Phys. J. C 22 (2001) 563.

8. A. Freitas, Ph.D. thesis, Hamburg (2002), DESYTHESIS-2002-023.

9. A. Freitas and A. v. Manteuffel, in Proc. of the 10th International Conference on Supersymmetry and Unification of Fundamental Interactions (SUSY02), Hamburg, Germany (2002), [hep$\mathrm{ph} / 0211105]$.

10. A. Freitas, A. v. Manteuffel and P. M. Zerwas, in preparation.

11. J. Guasch, W. Hollik and J. Solà, Phys. Lett. B 510 (2001) 211; hep-ph/0207364.

12. A. Freitas and D. Stöckinger, hep-ph/0205281, to be published in Phys. Rev. D; in Proc. of the 10th International Conference on Supersymmetry and Unification of Fundamental Interactions (SUSY02), Hamburg, Germany (2002), [hep-ph/0210372].

13. R. K. Ellis, D. A. Ross and A. E. Terrano, Nucl. Phys. B 178 (1981) 421; K. Fabricius, I. Schmitt, G. Kramer and G. Schierholz, Z. Phys. C 11 (1981) 315; J. A. Vermaseren, K. J. Gaemers and S. J. Oldham, Nucl. Phys. B 187 (1981) 301; Z. Kunszt, Phys. Lett. B 99 (1981) 429.

14. M. M. Weber, Ph.D. thesis, Hamburg (2001), DESY-THESIS-2002-003; M. Maniatis, Ph.D. thesis, Hamburg (2002), DESY-THESIS-2002007; A. Brandenburg, M. Maniatis and M. M. Weber, in Proc. of the 10th International Conference on Supersymmetry and Unification of Fundamental Interactions (SUSY02), Hamburg, Germany (2002), [hep-ph/0207278].

15. T. Tsukamoto, K. Fujii, H. Murayama, M. Yamaguchi and Y. Okada, Phys. Rev. D 51 (1995) 3153.

16. H. U. Martyn and G. A. Blair, in Proc. of the International Workshop on Linear Colliders, Sitges, Spain (1999), [hep-ph/9910416]; H. U. Martyn, contribution to Workshop on Physics at TeV Colliders, Les Houches, France (1999), [hep$\mathrm{ph} / 0002290]$.

17. M. Dima et al., Phys. Rev. D 65 (2002) 071701.

18. A. Freitas, D. J. Miller and P. M. Zerwas,
Eur. Phys. J. C 21 (2001) 361; A. Freitas and D. J. Miller, in Proc. of the APS/DPF/DPB Summer Study on the Future of Particle Physics (Snowmass 2001) eds. R. Davidson and C. Quigg, [hep-ph/0111430].

19. G. A. Blair, W. Porod and P. M. Zerwas, Phys. Rev. D 63 (2001) 017703; hep-ph/0210058; P. M. Zerwas et al., these proceedings [hep$\mathrm{ph} / 0211076]$.

20. H. Baer, C. Balazs, S. Hesselbach, J. K. Mizukoshi and X. Tata, Phys. Rev. D 63 (2001) 095008.

21. A. Datta and A. Djouadi, hep-ph/0111466; A. Datta, A. Djouadi and M. Mühlleitner, hep$\mathrm{ph} / 0204354$.

22. M. M. Nojiri, Phys. Rev. D 51 (1995) 6281.

23. E. Boos, H. U. Martyn, G. Moortgat-Pick, M. Sachwitz, A. Vologdin and P. M. Zerwas, in preparation; E. Boos, H. U. Martyn, G. MoortgatPick, M. Sachwitz and A. Vologdin, in Proc. of the 10th International Conference on Supersymmetry and Unification of Fundamental Interactions (SUSY02), Hamburg, Germany (2002), [hep-ph/0211040].

24. A. Bartl, K. Hidaka, T. Kernreiter and W. Porod, Phys. Lett. B 538 (2002) 137; hep-ph/0207186.

25. B. Ananthanarayan, S. D. Rindani and A. Stahl, hep-ph/0204233.

26. R. Keranen, A. Sopczak, H. Nowak and M. Berggren, Eur. Phys. J. direct C 7 (2000) 1.

27. H. Nowak and A. Sopczak, contributed paper to the ICHEP' 02 conference, ABS614.

28. K. Hidaka and A. Bartl, Phys. Lett. B 501 (2001) 78.

29. A. Bartl et al., Phys. Rev. D59 (1999) 115007. 